\title{
HIGH PRESSURE FAR-INFRARED MAGNETOOPTICAL STUDIES OF Sn AND S DONORS IN GaAs
}

\author{
J.E. DMOCHOWSKI \\ Institute of Physics, Polish Academy of Sciences \\ 02-668 Warszawa, Al. Lotników 32/46, Poland \\ and \\ Imperial College of Science, Technology and Medicine \\ Prince Consort Road, London, SW7 2BZ, UK \\ P.D. Wang and R.A. Stradling \\ Imperial College of Science, Technology and Medicine \\ Prince Consort Road, London, SW7 2BZ, UK
}

\begin{abstract}
The diamond anvil cell technique is applied for magneto-optical far-infrared transmission experiments with LPE grown GaAs:Sn. The $1 s-2 p(+)$ intra-shallow- $\Gamma$-donor transition is investigated as a function of high hydrostatic pressure for Sn and residual S donors. Both donors are shallow up to $30 \mathrm{kbar}$. Above this pressure both donors become deep and shallow donor absorption is persistently bleached due to deep non-metastable (non DX-like) states of the donors entering the gap of GaAs.
\end{abstract}

PACS numbers: 71.55.Eq, 78.50.Ge

The properties of donor impurities in III-V semiconductor compounds and alloys remain one of the most intensely discussed subjects. The final conclusion, regarding the origin of observed metastability effects, so-called DX-like behaviour, and possible mechanisms triggering shallow-deep instability for donor impurities has not been reached. It has been demonstrated experimentally [1-4] and theoretically confirmed [5-7] that two different mechanisms can be responsible for transformation of shallow donor into deep non-hydrogenic defect:

i) the impurity undergoes local lattice rearrangement upon capturing electron (or two electrons) forming DX-like state responsible for metastability effects;

ii) the electronic wave function of substitutional impurity "collapses" strongly localized electronic state is formed by local potential of substitutional 
impurity, without lattice relaxation. So, this shallow-deep transition has non-metastable character.

The experimental evidence for the latter transition has been demonstrated for Ge and Si donors in pressurized GaAs [2-4, 8]. We present here experiments in which we investigate shallow-deep transition for $\mathrm{Sn}$ and $\mathrm{S}$ donors in high-purity GaAs at high pressure. We employ developed for this purpose diamond anvil cell technique for far-infrared magneto-optical transmission experiments using far-infrared molecular laser [9]. The absorption due to $1 s-2 p(+)$ hydrogenic intra-shallow- $\Gamma$-donor transition is monitored as a function of pressure. The transmission observed at three pressures - ambient (1 bar), $19 \mathrm{kbar}$ and $30 \mathrm{kbar}$ - for LPE grown Sn doped high purity sample is presented in Fig. 1. The fine structure

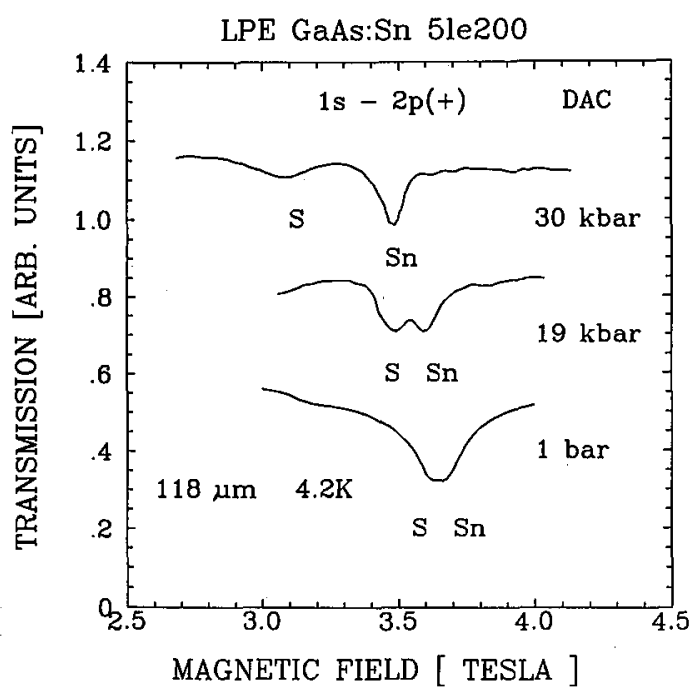

Fig. 1. Pressure dependence of $1 s-2 p(+)$ absorption due to $S$ and Sn donors in LPE grown GaAs:Sn, observed with the diamond anvil cell technique. Above $30 \mathrm{kbar}$ no absorption is observed either in darkness or under strong white light illumination.

of the $1 s-2 p(+)$ transition, due to different "chemical shifts" for two donor species - tin and residual sulphur - present in the sample, can be resolved. The existence of shallow donor absorption up to $30 \mathrm{kbar}$ independent on the experimental conditions, i.e. cooling in darkness or exposure to white light, demonstrates that shallow hydrogenic donor state is the ground state of both donor impurities in this pressure range. Above $30 \mathrm{kbar}$, however, no absorption is observed, either in darkness or under strong white light illumination. It has been demonstrated for Si donor that this phenomenon is related to appearance in the gap of deep impurity state which can be observed in photoluminescence, i.e. under continuous strong illumination [10]. This state does not follow upon applying pressure the lowest conduction band and persistently captures electrons from the shallow state. The deep state can be identified with antibonding localized donor state with $A_{1}$ symmetry, and not with DX-like state. The deep state reported for photoluminescence experiments with GaAs:S which enters the gap close to $30 \mathrm{kbar}$ [10], has all attributes of such a 
state. This state cannot be DX-like state, as suggested by the authors of Ref. [10]. They do realize, that population of this state is not sensitive to illumination, so it does not possess metastable properties. One can thus conclude that for $\mathrm{Si}$ and $S$ donors the shallow-deep transition without lattice relaxation occurs close to 30 $\mathrm{kbar}$. The evidence for analogous transition for $\mathrm{Sn}$ donor close to $30 \mathrm{kbar}$ is given in far-infrared experiments. However, the existence of unrelaxed deep state for $\mathrm{Sn}$ donors is to be confirmed with photoluminescence experiments.

The second interesting result of our high pressure investigations is much better resolution of "chemical" fine structure of $1 s-2 p(+)$ transition at high pressures. This can be related to increase of "chemical shift" of the ground $1 s$ state with pressure for both impurities. The larger is "chemical shift" at ambient pressure, the bigger increase with pressure (Fig. 2). This behaviour has been theoretically

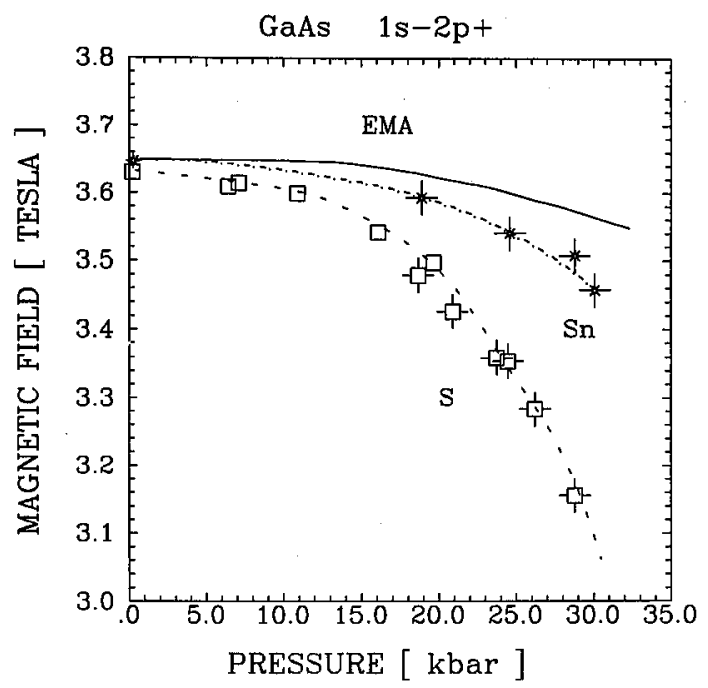

Fig. 2. The magnetic field position against pressure of the absorption peaks observed at $118 \mu \mathrm{m}$ wavelength due to $\mathrm{S}$ and $\mathrm{Sn}$ donors. EMA line is effective mass theory prediction which takes into account pressure dependence of effective mass and dielectric constant [8]. The theory is corrected for "chemical shift" characteristic of Sn donor at ambient pressure, which is assumed to be pressure independent.

predicted in [11]. It corresponds to increase with pressure of the density of $1 s$ electronic wave function at the impurity core and consequently stronger interaction of electron with local potential.

\section{References}

[1] Z. Wasilewski, R.A. Stradling, S. Porowski, Solid State Commun. 57, 123 (1986).

[2] J.E. Dnuchowski, Z. Wasilewski, R.A. Stradling, in Shallow Impurities in Semiconductors, Ed. G. Davies, Mater. Sci. Forum 65-66, Trans-Tech, Zürich 1991, p. 449. 
[3] J.E. Dmochowski, P.D. Wang, R.A. Stradling, Semic. Sci. Technol. 6, 118 (1991).

[4] J.E. Dmochowski, R.A. Stradling, P.D. Wang, S.N. Holmes, M. Li, B.D. McCombe, B. Weinstein, Semic. Sci. Technol. 6, 476 (1991).

[5] D.J. Chadi, K.J. Chang, Phys. Rev. Lett. 61, 873 (1988); Phys. Rev. B 39, 10063 (1989).

[6] J. Dabrowski, M. Scheffler, R. Strehlow, in The Physics of Semiconductors, Proc. 20th ICPS, Eds. E.M. Anastassakis, J.D. Joannopoulos, Vol. 1, World Scientific, Singapore 1990, p. 489.

[7] E. Yamaguchi, K. Shiraishi, T. Ohno, ibid. p. 501.

[8] Z. Wasilewski, R.A. Stradling, Semic. Sci. Technol. 1, 264 (1986).

[9] J.E. Dmochowski, P.D. Wang, R. Wooley, P.C. Klipstein, R.A. Stradling, in Proc. 4th Int. Conf. "High Pressure in Semiconductor Physics", Porto Carras 1990, Eds. D.S. Kyriakos, A.E. Valassiades, Thessaloniki 1990, p. 148.

[10] X. Liu, L. Samuelson, M.-E. Pistol, M. Gerling, S. Nilsson, Phys. Rev. B 42, 11791 (1990).

[11] W. Trzeciakowski, J. Krupski, Solid State Commun. 44, 1491 (1982). 